Available online at GSC Online Press Directory

GSC Biological and Pharmaceutical Sciences

e-ISSN: 2581-3250, CODEN (USA): GBPSC2

Journal homepage: https://www.gsconlinepress.com/journals/gscbps

(RESEARCH ARTICLE)

\title{
Contribution of garlic for improving the cytoprotective effect of mesna against cyclophosphamide toxicity in rats
}

\author{
Tahoun Enas Abdelaziz ${ }^{1}$, El- Borai Nermeen Borai ${ }^{2, *}$, Khalifa Hanem Kamal ${ }^{3}$ and El- Gendy Hanem ${ }^{4}$ \\ ${ }^{1}$ Department of Pathology, Faculty of Veterinary medicine, University of Sadat City, Egypt. \\ 2 Department of Forensic Medicine and Toxicology, Faculty of Veterinary medicine, University of Sadat City, Egypt. \\ ${ }^{3}$ Department of Biochemistry and Chemistry of Nutrition, Faculty of Veterinary medicine, University of Sadat City, Egypt. \\ ${ }^{4}$ Department of Pharmacology, Faculty of Veterinary medicine, University of Sadat City, Egypt.
}

Publication history: Received on 16 May 2019; revised on 27 May 2019; accepted on 03 June 2019

Article DOI: https://doi.org/10.30574/gscbps.2019.7.3.0089

\begin{abstract}
Cyclophosphamide (CYP), an oxazophosphorine- alkylating agent, is a widely used drug for treatment of neoplastic and severe autoimmune diseases. Adverse effects of CYP restrict its therapeutic benefits. Mesna (MS), a thiol compound, is used as a protective agent against hemorrhagic cystitis induced by CYP. This study aimed to investigate the potential role of raw garlic homogenate $(\mathrm{RGH})$ for improving the protective effect of mesna against CYP toxicity in rats. Thirty male albino rats were divided into five equal groups. Control group, CYP group (a single i.p. dose, $200 \mathrm{mg} / \mathrm{kg}$ b.wt.), CYP+MS group (a total dose of $120 \mathrm{mg} / \mathrm{kg}$ b.wt. in three equal doses, i.p.), CYP+RGH group (500 mg/kg b.wt., orally, once daily 5 days before and after CYP injection) and CYP+MS+RGH group. CYP induced hematological changes, including significant reduction of RBCs, Hb, PCV with thrombocytopenia and leukocytopenia. Also, significant increase in serum MDA content concomitantly with significant reduction in TAC was recorded. This was associated with histopathological alterations in the examined tissues. However, mesna and/or garlic improved the recorded hematological, biochemical and histopathological alterations induced by CYP with marked improvement using their combination. In conclusion, garlic supplementation in combination with mesna could be of a great value to introduce therapeutic strategies for patients undertaking cyclophosphamide therapy.
\end{abstract}

Keywords: Cyclophosphamide; Garlic; Mesna; Hematology; Histopathology

\section{Introduction}

Cyclophosphamide (CYP) is an alkylating agent and widely used anti-neoplastic drug for the treatment of malignant lymphomas, myeloma, leukemia, neuroblastoma, adenocarcinoma, retinoblastoma and breast carcinoma [1] and as a preparatory treatment for bone marrow transplantation [2]. Because of its immunosuppressive activity, it is also frequently used as an immunosuppressive agent for organ transplantation [3] and for treating autoimmune diseases [4]. Also, it used for treatment of amyloidosis, idiopathic nephritis, severe rheumatoid arthritis and multiple sclerosis $[5,6]$. Most of these treatment regimens require high doses of CYP that are associated with adverse effects such as hemorrhagic cystitis; hematological, immunological, cardiac, hepatic and renal toxicities [7-11].

Mesna (sodium-2-mercaptoethane-sulfonate) is a thiol compound that used clinically as a protective agent against the toxicity of chemotherapy and as a regional detoxificant to reduce the incidence of haemorrhagic cystitis and haematuria in patients received CYP [12]. Furthermore, mesna exerts anti-inflammatory, anti-oxidative and anti-apoptotic properties [13]. Mesna is not able to perfectly counteract the side effect of CYP, and studies since its discovery have investigated the use of alternatives to increase its efficacy. Natural herbs have been used to treat various diseases and

\footnotetext{
${ }^{*}$ Corresponding author

E-mail address: nermeenborai@yahoo.com
} 
provided as chemotherapeutic agents to protect against toxic side effects mainly due to their antioxidant activities [14, 15].

Garlic (Allium sativum) is a worldwide traditional food and dietary supplement. Nowadays, several garlic preparations including fresh or aged garlic extracts, garlic powder and garlic oil are used as a remedy for a variety of diseases. Raw garlic homogenate is the most frequently used garlic preparation for scientific studies, because it is the most consumed garlic [16].

Garlic contains at least 33 sulfur compounds, amino acids, minerals and vitamins [17]. The chemical constituents of garlic have been recommended for treatment of cardiovascular disease, cancer, diabetes, blood pressure, atherosclerosis and hyperlipidaemia. Moreover, the sulfur compounds of garlic have antioxidant actions by scavenging ROS, enhancing cellular antioxidant enzymes and increasing glutathione in the cells [18]. Cyclophosphamide is able to generate ROS and induce oxidative damage. Thus, strategies to minimize the side effects of chemotherapeutic agents while preserving their efficacy are needed. In the light of this, the current study aimed to investigate the potential role of raw garlic homogenate for improving the protective effect of mesna against cyclophosphamide toxicity in rats.

\section{Material and methods}

\subsection{Experimental Animals}

A total of thirty healthy male albino rats (195-200 g) were purchased from Al-Zyade Experimental Animals Production Center, Giza, Egypt. Animals were housed in polypropylene cages with mesh wire tops and kept in a natural ventilated room $26 \pm 3{ }^{\circ} \mathrm{C}, 45-60 \%$ relative humidity, natural daily dark/light cycle, provided with standard commercial diet and clean tap water ad libitum. Rats were acclimatized to the laboratory environmental conditions for 2 weeks before the beginning of the experiment.

\subsection{Chemicals}

Cyclophosphamide (Endoxan ${ }^{\circledR} 200 \mathrm{mg}$, Baxter Oncology GmbH, Germany), was dissolved in $5 \mathrm{ml}$ sterile distilled water to a concentration of $40 \mathrm{mg} / \mathrm{ml}$ immediately before treatment and mesna (Uromes ${ }^{\circledR} 400 \mathrm{mg} / 4 \mathrm{ml}$, EMIC United Company) were purchased from local pharmacy. Diagnostic kits for assaying serum biochemical parameters were purchased from Biodiagnostic Company, Dokki, Giza, Egypt. Other utilized chemicals were of analytical grade and commercially available.

\subsection{Preparation of raw garlic homogenate}

Fresh garlic was collected from local market. The cloves were cleaned, peeled, sliced, ground into a paste and a homogenate was made in distilled water. Freshly prepared extract at concentration of $0.2 \mathrm{mg} / \mathrm{ml}$ (corresponding to 500 $\mathrm{mg} / \mathrm{kg}$ b.wt.) was orally administrated to rats within $30 \mathrm{~min}$ after preparation [19].

\subsection{Experimental design and animal grouping}

Rats were weighed and randomly allocated into five equal groups. Control group, rats received only the standard diet and tap water. CYP group, rats received a single i.p. dose, $200 \mathrm{mg} / \mathrm{kg} \mathrm{b.wt.} \mathrm{at} 6^{\text {th }}$ day of the experiment [20]. CYP+MS group, rats received CYP and mesna as a total dose of $120 \mathrm{mg} / \mathrm{kg}$ b.wt., divided into three i.p. equal doses, $20 \mathrm{~min}$ before and 4, 8 hours after CYP injection [21]. CYP+RGH group, rats received CYP and RGH oral administration (500 mg/kg b.wt.), once daily 5 days before and after CYP injection [19]. CYP+MS+RGH, rats received CYP, MS and RGH as previously mentioned (Table 1). 
Table 1: The experimental design and animal grouping.

\begin{tabular}{|c|c|c|c|c|c|c|c|c|}
\hline \multirow[b]{3}{*}{ Group } & \multicolumn{7}{|c|}{ Days of experiment } & \multirow{8}{*}{ 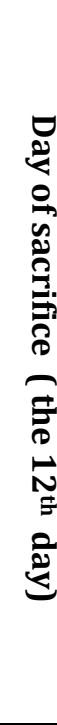 } \\
\hline & \multirow{2}{*}{$\begin{array}{c}\begin{array}{c}\text { Before CYP } \\
\text { injection }\end{array} \\
5 \text { days } \\
\left(1^{\text {st }}-5^{\text {th }} \text { day }\right)\end{array}$} & \multicolumn{5}{|c|}{$\begin{array}{c}\text { Day of CYP injection at the } \\
6^{\text {th }} \text { day of the experiment }\end{array}$} & \multirow{2}{*}{$\begin{array}{c}\begin{array}{c}\text { After CYP } \\
\text { injection }\end{array} \\
5 \text { days } \\
\left(7^{\text {th }}-11^{\text {th }} \text { day }\right) \\
\end{array}$} & \\
\hline & & $-60 \min$ & $-20 \min$ & 0 & $\begin{array}{c}+4 \\
\mathrm{~h}\end{array}$ & $+8 \mathrm{~h}$ & & \\
\hline Control & --------- & ------ & ------ & ----- & --- & ---- & ----------- & \\
\hline CYP & ----------- & ----- & ----- & CYP & --- & ----- & ------------ & \\
\hline $\mathrm{CYP}+\mathrm{MS}$ & --------- & ------ & MS & CYP & MS & MS & ---------- & \\
\hline $\mathrm{CYP}+\mathrm{RGH}$ & $\mathrm{RGH}$ & $\mathrm{RGH}$ & ----- & CYP & $\begin{array}{l}--- \\
--\end{array}$ & --- & $\mathrm{RGH}$ & \\
\hline $\mathrm{CYP}+\mathrm{MS}+\mathrm{RGH}$ & $\mathrm{RGH}$ & $\mathrm{RGH}$ & MS & CYP & MS & MS & $\mathrm{RGH}$ & \\
\hline
\end{tabular}

\subsection{Collection of samples}

At the $12^{\text {th }}$ of the experiment, rats were fasted overnight, anaesthetized and blood samples were collected from the medial canthus of the eye. Blood samples were divided into two parts. The first part was collected on EDTA for hematological analysis, while the other part was used for serum collection and was stored at $-20 \stackrel{\circ}{\circ}$ until be used for biochemical analysis. Immediately after blood collection, rats were rapidly sacrificed and tissue specimens from the urinary bladder, liver, kidney, heart and spleen were collected and fixed in $10 \%$ neutral buffered formalin solution for histopathological examination.

\subsection{Hematological examination}

Estimation of red blood cells (RBCs) count, hemoglobin (Hb) concentration, packed cell volume (PCV) \%, total (TLC) and differential leukocytic counts were measured according to the routine hematological procedures described by Weiss and Wardrop [22]. Mean corpuscular volume (MCV) was calculated as PCV divided by red cell count and multiplied by 10. Mean corpuscular hemoglobin (MCH) was calculated as hemoglobin divided by red cell count and multiplied by 10 , while mean corpuscular hemoglobin concentration (MCHC) was calculated as hemoglobin divided by PCV multiplied by 100.

\subsection{Serum oxidant/ antioxidant biomarkers}

Serum Malondialdehyde (MDA) content and total antioxidant capacity (TAC) were estimated according to Ohkawa et al. [23] and Koracevic et al. [24], respectively, using commercial kits.

\subsection{Histopathological examination}

The formalin fixed specimens were trimmed, washed, dehydrated in ascending grades of ethyl alcohol, cleared in methyl benzoate and embedded in paraffin after having completed the routine follow-up steps. Sections at 3-5 $\mu$ sections were obtained from urinary bladder, liver, kidney, heart and spleen using microtome (LEICA RM 2135) and stained by hematoxylin and eosin (H\&E) stain for light microscopical investigation according to Bancroft and Gamble [25]. Photos were taken using digital camera (LEICA DMLB Germany).

\subsection{Statistical analysis}

Values are presented as mean \pm standard error. Statistical analysis of data was carried out using analysis of variance by one-way ANOVA test followed by Duncan multiple comparison tests. All data were statistically analyzed using statistical software program SPSS (Statistical package for Social Sciences) Version 16 released on 2007. 


\section{Results}

\subsection{Effects on animals' general condition}

Control rats did not exhibit any clinical manifestations. However, dullness, depression, lethargy, rough hair coat, dehydration were the observed signs of CYP- treated group. Other groups exhibited less sever clinical manifestations.

\subsection{Effect on hematological parameters}

Compared to control group, rats of the CYP- treated group revealed significant $(\mathrm{P}<0.05)$ decreases of RBCs count, Hb, PCV without any significant changes in MCV, MCH and MCHC levels along with thrombocytopenia. Moreover, CYP induced significant reduction in WBCs, neutrophils and lymphocytes with insignificant reduction in eosiniphils, basophils and monocytes. However, administration of mesna and/or garlic improved these values with marked improvement in their combination (Table 2).

Table 2 Hematological parameters in control and different treated groups

\begin{tabular}{llllll}
\hline Parameters & Control & CYP & CYP + MS & CYP + RGH & CYP+MS+RGH \\
\hline RBCs $\left(\mathrm{x} 10^{6} / \mu \mathrm{l}\right)$ & $8.39 \pm 0.31^{\mathrm{a}}$ & $4.70 \pm 0.19^{\mathrm{d}}$ & $6.73 \pm 0.27^{\mathrm{b}}$ & $5.57 \pm 0.22^{\mathrm{c}}$ & $7.70 \pm 0.25^{\mathrm{a}}$ \\
PCV $(\%)$ & $44.87 \pm 3.56^{\mathrm{a}}$ & $27.59 \pm 1.70^{\mathrm{c}}$ & $39.05 \pm 1.65^{\mathrm{ab}}$ & $33.99 \pm 1.08^{\mathrm{bc}}$ & $42.82 \pm 3.11^{\mathrm{a}}$ \\
Hb (g/dl) & $14.30 \pm 0.61^{\mathrm{a}}$ & $9.67 \pm 0.44^{\mathrm{d}}$ & $12.10 \pm 0.41^{\mathrm{bc}}$ & $10.80 \pm 0.25^{\mathrm{cd}}$ & $12.83 \pm 0.57^{\mathrm{ab}}$ \\
MCV (fl) & $53.29 \pm 2.39^{\mathrm{b}}$ & $55.49 \pm 2.26^{\mathrm{ab}}$ & $61.07 \pm 1.68^{\mathrm{a}}$ & $58.05 \pm 0.49^{\mathrm{ab}}$ & $58.55 \pm 1.33^{\mathrm{ab}}$ \\
MCH (pg) & $16.71 \pm 0.91^{\mathrm{b}}$ & $19.41 \pm 0.52^{\mathrm{ab}}$ & $18.03 \pm 0.78^{\mathrm{ab}}$ & $20.68 \pm 1.68^{\mathrm{a}}$ & $17.04 \pm 0.31^{\mathrm{b}}$ \\
MCHC $(\%)$ & $30.31 \pm 2.79$ & $31.79 \pm 0.28$ & $31.05 \pm 1.18$ & $35.48 \pm 3.76$ & $32.11 \pm 1.69$ \\
Platelets $\left(\mathrm{x} 10^{3} / \mu \mathrm{l}\right)$ & $491 \pm 9.70^{\mathrm{a}}$ & $196 \pm 9.40^{\mathrm{d}}$ & $332 \pm 9.23^{\mathrm{c}}$ & $397 \pm 8.84^{\mathrm{b}}$ & $472 \pm 8.04^{\mathrm{a}}$ \\
TLC $\left(\mathrm{x} 10^{3} / \mu \mathrm{l}\right)$ & $9.06 \pm 0.19^{\mathrm{a}}$ & $3.46 \pm 0.32^{\mathrm{d}}$ & $5.50 \pm 0.58^{\mathrm{c}}$ & $6.86 \pm 0.45^{\mathrm{b}}$ & $8.64 \pm 0.27^{\mathrm{a}}$ \\
Neutrophils $\left(\mathrm{x} 10^{3} / \mu \mathrm{l}\right)$ & $3.20 \pm 0.095^{\mathrm{a}}$ & $1.55 \pm 0.13^{\mathrm{c}}$ & $1.79 \pm 0.16^{\mathrm{c}}$ & $2.40 \pm 0.23^{\mathrm{b}}$ & $2.86 \pm 0.18^{\mathrm{ab}}$ \\
Lymphocytes $\left(\mathrm{x} 10^{3} / \mu \mathrm{l}\right)$ & $5.30 \pm 0.04^{\mathrm{a}}$ & $1.59 \pm 0.15^{\mathrm{c}}$ & $2.91 \pm 0.48^{\mathrm{b}}$ & $3.72 \pm 0.35^{\mathrm{b}}$ & $4.65 \pm 0.23^{\mathrm{a}}$ \\
Eosinophils $\left(\mathrm{x} 10^{3} / \mu \mathrm{l}\right)$ & $0.18 \pm 0.01^{\mathrm{bc}}$ & $0.03 \pm 0.02^{\mathrm{c}}$ & $0.73 \pm 0.04^{\mathrm{a}}$ & $0.35 \pm 0.21^{\mathrm{abc}}$ & $0.62 \pm 0.23^{\mathrm{ab}}$ \\
Basophils $\left(\mathrm{x} 10^{3} / \mu \mathrm{l}\right)$ & $0.02 \pm 0.003^{\mathrm{b}}$ & $0.21 \pm 0.003^{\mathrm{b}}$ & $0.01 \pm 0.006^{\mathrm{b}}$ & $0.01 \pm 0.006^{\mathrm{b}}$ & $0.05 \pm 0.01^{\mathrm{a}}$ \\
Monocytes $\left(\mathrm{x} 10^{3} / \mu \mathrm{l}\right)$ & $0.35 \pm 0.06^{\mathrm{ab}}$ & $0.28 \pm 0.09^{\mathrm{ab}}$ & $0.20 \pm 0.05^{\mathrm{b}}$ & $0.38 \pm 0.05^{\mathrm{ab}}$ & $0.46 \pm 0.02^{\mathrm{a}}$ \\
\hline
\end{tabular}

Values are presented as mean \pm SE $(n=5)$. Means with different superscripts in the same raw are significantly different at $(\mathrm{P}<0.05)$.

CYP: Cyclophosphamide; MS: Mesna; RGH: Raw garlic homogenate. RBCs, red blood cell count; Hb, hemoglobin; PCV, packed cell volume; MCV, mean corpuscular volume; $\mathrm{MCH}$, mean corpuscular hemoglobin; $\mathrm{MCHC}$, mean corpuscular hemoglobin concentration; TLC, total leukocyte count.

\subsection{Oxidant/ antioxidant biomarkers}

Administration of CYP to rats significantly increased $(\mathrm{P}<0.05)$ serum MDA content concomitantly with reduction in TAC in compared to control group. Rats administered mesna or garlic significantly abolished these changes and restored the normal control values in rats administered a combination of MS and RGH (Table 3).

Table 3 Serum oxidant/ antioxidant biomarkers in control and different treated groups

\begin{tabular}{lccccc}
\hline Parameters & Control & CYP & CYP + MS & CYP + RGH & CYP+ MS+ RGH \\
\hline MDA (nmol/ml) & $4.23 \pm 0.17^{\mathrm{b}}$ & $8.47 \pm 0.29^{\mathrm{a}}$ & $6.57 \pm 0.39^{\mathrm{c}}$ & $6.34 \pm 0.27^{\mathrm{c}}$ & $4.92 \pm 0.11^{\mathrm{b}}$ \\
TAC (mmol/ml) & $1.48 \pm 0.17^{\mathrm{a}}$ & $0.62 \pm 0.06^{\mathrm{b}}$ & $0.92 \pm 0.05^{\mathrm{c}}$ & $0.95 \pm 0.02^{\mathrm{c}}$ & $1.41 \pm 0.12^{\mathrm{a}}$ \\
\hline
\end{tabular}

Values are presented as mean \pm SE $(n=5)$. Means with different superscripts in the same raw are significantly different at $(\mathrm{P}<0.05)$. CYP: Cyclophosphamide; MS: Mesna; RGH: Raw garlic homogenate. MDA, Malondialdehyde; TAC, total antioxidant capacity. 


\subsection{Histopathological findings}

\subsubsection{Microscopic alterations of urinary bladder}

The transitional epithelium and basement membrane that separates the epithelium from the underlying lamina propria of the bladders were preserved in control group (Fig.1 A). In CYP-treated rats, there were epithelial desquamation, reactive hyperplasia of urinary epithelium, dilatation and congestion of bladder vessels with leukocyte infiltration, hemorrhage and edema in the connective tissues that constituted the lamina propria (Fig.1 B1,2). However, rats administered CYP and mesna showed only mild connective tissue edema and the uroepithelium thickness was diminished and no uroepithelial erosion or ulceration was observed (Fig.1 C). Similarly, CYP+RGH- treated rats showed that uroepithelium thickness was preserved with mild uroepithelial desquamation and mild congestion of blood vessels without connective tissue edema (Fig.1 D). Nearly normal urinary bladder histology was observed in CYP+MS+RGHtreated rats (Fig.1 E).
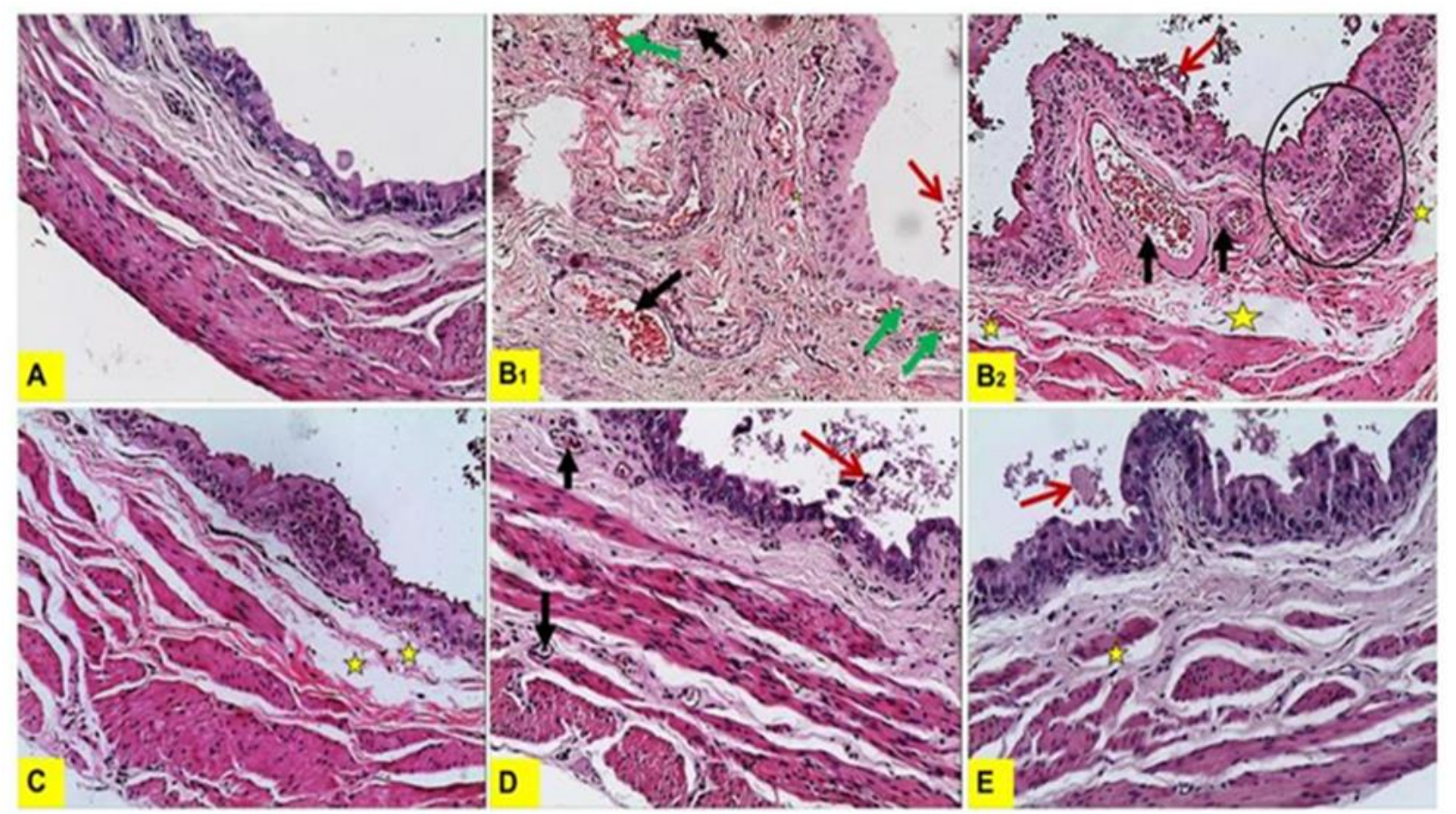

Figure 1 Photomicrographs of urinary bladder sections of rats in different groups (H\&E stain $\mathrm{X}_{10}$ ) (star: edema, red arrow: uroepithelial desquamation, black arrow: congestion, green arrow: hemorrhage, circle: reactive hyperplasia of urinary epithelium) A: Control group showing normal histology. B1, 2: CYP group showing uroepithelial desquamation, reactive hyperplasia of urinary epithelium, dilatation and congestion of bladder vessels with leukocyte infiltration and edema. C: CYP+MS group showing uroepithelial preservation and mild edema. D: CYP+RGH group showing uroepithelial preservation and mild congestion of blood vessels. E: CYP+MS+RGH group showing somewhat normal histology and uroepithelial preservation with mild edema.

\subsubsection{Microscopic alterations of liver}

Control group showing normal hepatic architecture (Fig. 2 A). However, CYP- treated rats showed diffuse granular degeneration of most hepatocytes, edema, congestion of central vein and hepatic sinusoids, inflammatory cells infiltration, hepatic cells with nuclear pyknosis, some apoptotic hepatic cells and multifocal areas of coagulative necrosis infiltrated by inflammatory cells. (Fig.2 B1, 2). Liver sections of CYP+MS- treated rats showed congestion of hepatic sinusoids, some hepatic cells with nuclear pyknosis (Fig.2 C). While, CYP+RGH- treated rats showed mild congestion of central vein and hepatic sinusoids and small area of coagulative necrosis infiltrated by some inflammatory cells. (Fig. 2 D). No histological change was observed in liver of CYP+MS+RGH-treated rats (Fig. 2 E). 

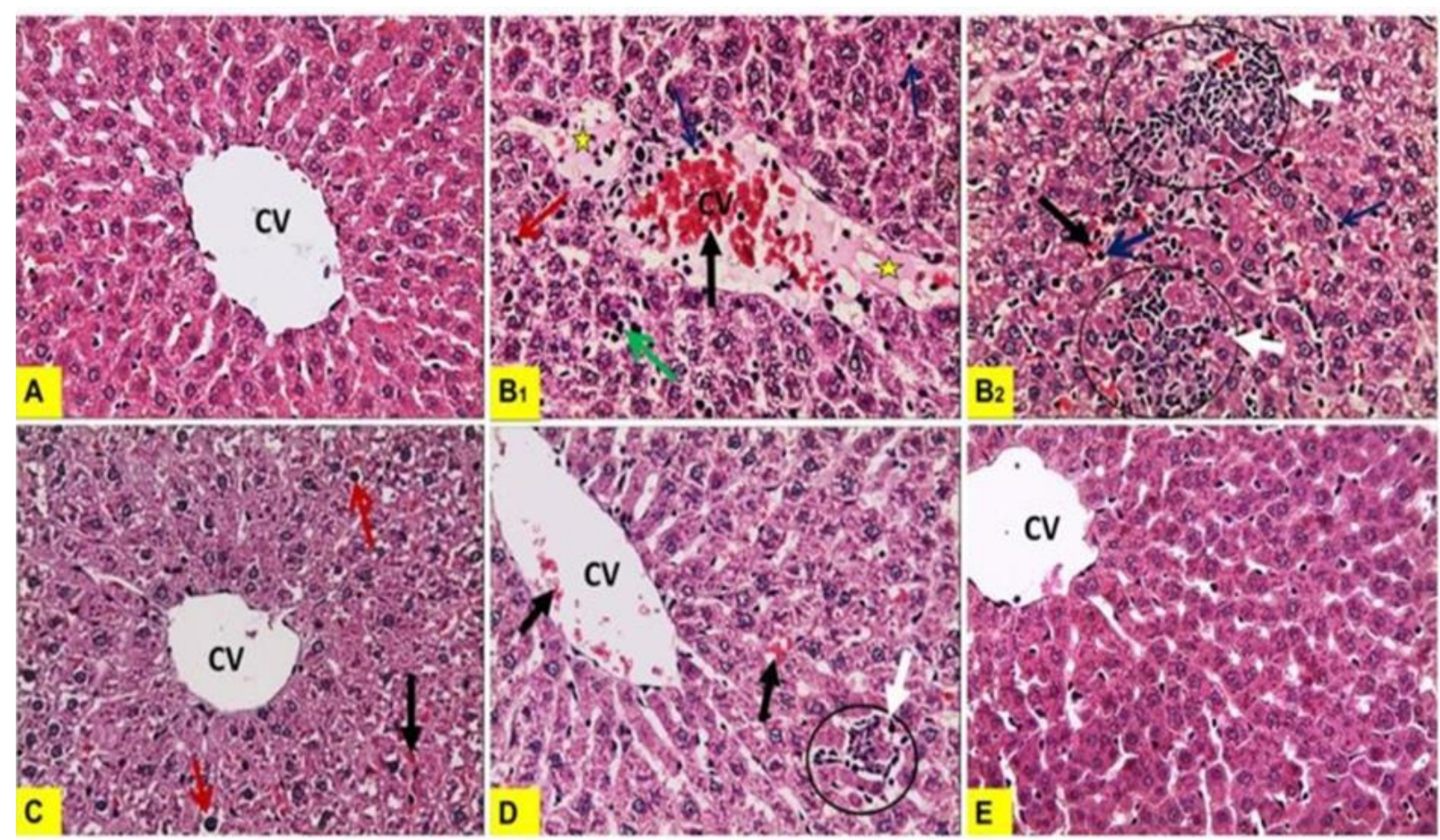

Figure 2 Photomicrographs of liver sections of rats in different groups (H\&E stain X20) (star: edema, black arrow: congestion, red arrow: nuclear pyknosis, blue arrow: inflammatory cells infiltration, green arrow: apoptotic hepatic cells, white arrow and circle: area of coagulative necrosis infiltrated by inflammatory cells) A: Control group showing normal tissue architecture of liver. B1,2: CYP group showing edema, congestion of central vein and hepatic sinusoids, inflammatory cells infiltration, hepatic cells with nuclear pyknosis, some apoptotic hepatic cells and multifocal areas of coagulative necrosis infiltrated by inflammatory cells. C: CYP+MS group showing congestion of hepatic sinusoids, some hepatic cells with nuclear pyknosis. D: CYP+RGH group showing mild congestion of central vein and hepatic sinusoids, small area of coagulative necrosis infiltrated by some inflammatory cells. E: CYP+MS+RGH group showing: somewhat normal histological structure of liver parenchyma.

\subsubsection{Microscopic alterations of kidney}

Normal renal tissue was observed in control group (Fig.3 A). CYP- treated rats showed interstitial edema, marked glomerular congestion, sever glomerular and interstitial hemorrhage, glomerular atrophy with nuclear pyknosis of glomerular cells, dilated renal tubules with cloudy swelling and intraluminal desquamated epithelial cells (Fig.3 B1,2). Kidney of CYP+MS- treated rats showed mild glomerular congestion, glomerular atrophy with nuclear pyknosis of glomerular cells, dilatation of some renal tubules with cloudy swelling (Fig.3 C). While, CYP+RGH-treated rats showed mild congestion of glomeruli, slight interstitial hemorrhage and most renal tubules were apparently normal (Fig.3 D). Somewhat normal intact tubules and glomerular tufts with no observable histological changes were observed in CYP+MS+RGH- treated rats (Fig.3 E).

\subsubsection{Microscopic alterations of heart}

Rats of control group showing normal myocardial muscles and interstitial tissue (Fig.4 A). Hearts of CYP-administrated rat showed loss of striation of myocardial muscles, hemorrhage, edema and lymphocytic infiltration between myocardial muscles in addition to myocardial necrosis and cardiomyolysis with granular appearance of sarcoplasm and myofibers (Fig.4 B1,2). Heart of CYP+MS (Fig.4 C) and CYP+RGH (Fig.4 D) groups showed mild hemorrhage, edema between myocardial muscles beside necrosis in some myocardial muscles were observed. CYP+MS+RGH- treated rats showed somewhat normal histology except single cell necrosis myocardial muscle (Fig.4 E). 

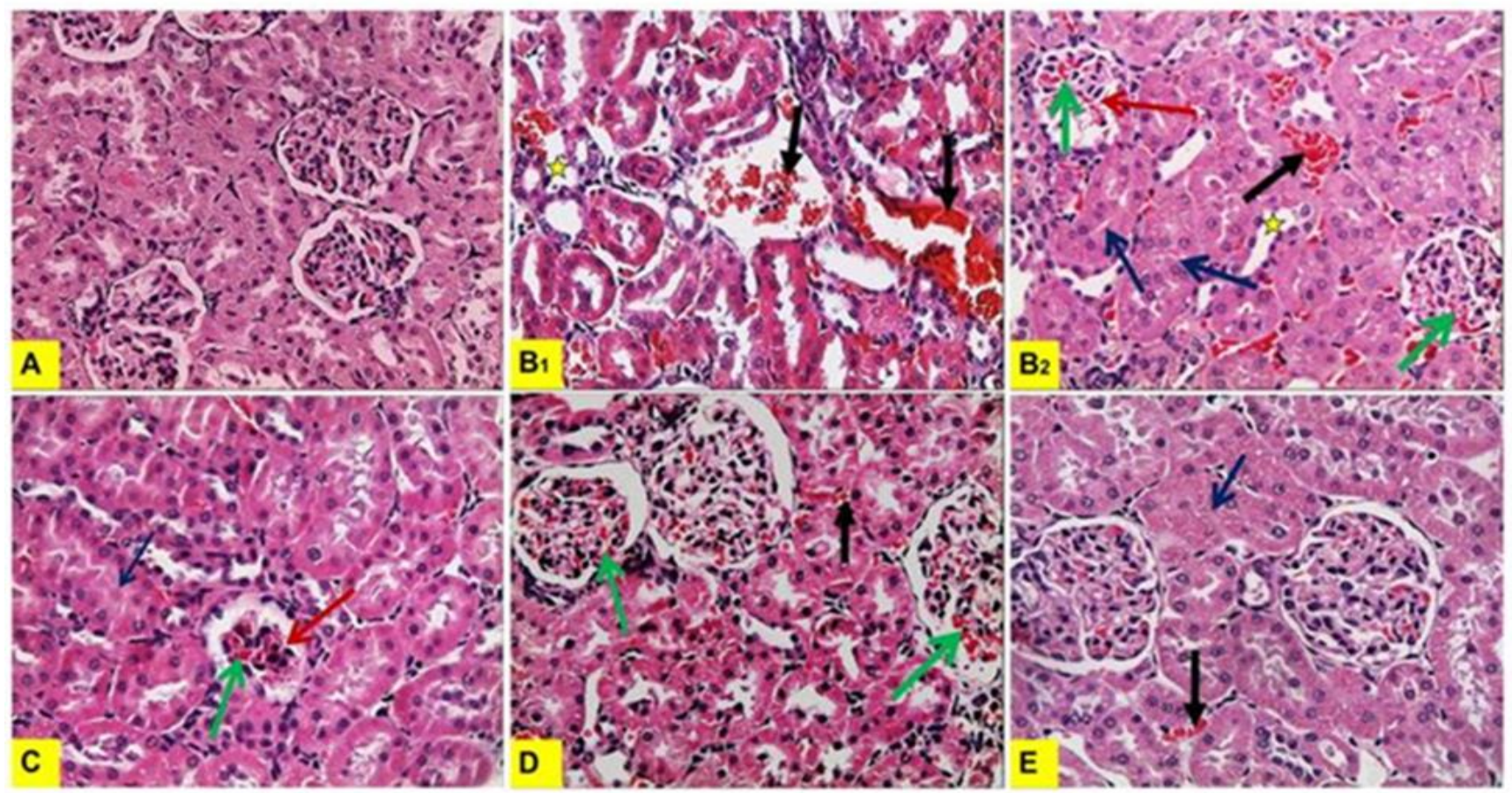

Figure 3 Photomicrographs of kidney sections of rats in different groups (H\&E stain X20) (star: edema, black arrow: hemorrhage, red arrow: glomerular atrophy with nuclear pyknosis of glomerular cells, blue arrow: dilated renal tubules with cloudy swelling) A: Control group showing normal tissue architecture of kidney. B1,2: CYP group showing glomerular congestion, glomerular and interstitial hemorrhage, glomerular atrophy with nuclear pyknosis of glomerular cells, dilated renal tubules with cloudy swelling, interstitial edema. C: CYP+MS group showing glomerular congestion, glomerular atrophy with nuclear pyknosis of glomerular cells, dilated renal tubules with cloudy swelling. D: $\mathrm{CYP}+\mathrm{RGH}$ group showing congestion of glomeruli, slight interstitial hemorrhage. E: CYP+MS+RGH group showing somewhat normal histological structure of renal tissue.
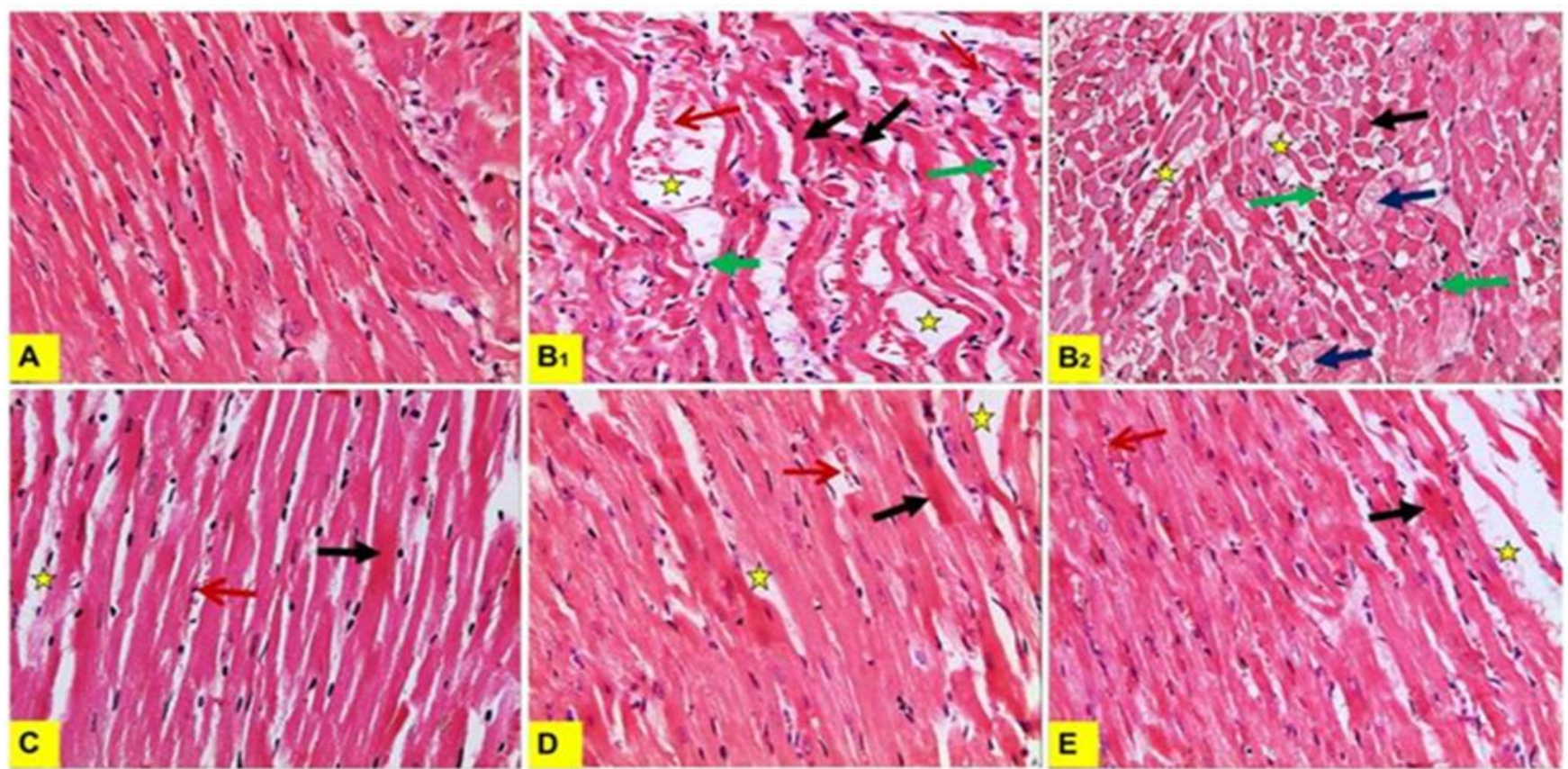

Figure 4 Photomicrographs of heart sections of rats in different groups (H\&E stain X20) (star: edema; red arrow: hemorrhage, green arrow: lymphocytic infiltration, black arrow: Zenker's necrosis, blue arrow: cardiomyolysis) A: Control group showing: Normal histology. B1,2: CYP group: B1 longitudinal section B2 cross section showing: loss of striation, hemorrhage, edema and lymphocytic infiltration between myocardial muscles and myocardial necrosis and cardiomyolysis with granular appearance of sarcoplasm and myofibers. C: CYP+MS group, D: CYP+RGH group showing 
mild hemorrhage, edema between myocardial muscles beside necrosis in some myocardial muscles. $\mathrm{E}$ : $\mathrm{CYP}+\mathrm{MS}+\mathrm{RGH}$ group showing: somewhat normal except single cell necrosis myocardial muscle.

\subsubsection{Microscopic alterations of spleen (Fig. 5):}

Histopathological examination in the spleen of control rats showed normal architecture. The splenic follicles (lymphatic nodules) in white pulp were big, intact with eccentric follicular arterioles and contain small deeply basophilic lymphocyte aggregation (Fig. 5 A). Several histopathological changes were recorded in spleen of CYP- treated group, including progressive loss of white pulp and relative increases in red pulp also marked loss in distinction between white and red pulps was noted, the lymphatic nodules were dispersed with decrease in its lymphocyte population and marked loss in the chromatin of their nuclei and most of the lymphocytes contained pyknotic nuclei, the reticular cells in the red pulp were increased in number and size and their nuclei were fragmented or necrotic and increase in the size of megakaryocytes with many nuclei were observed in CYP- treated rats (Fig. 5 B1,2). Little histopathological alterations and marked improvement in the splenic tissue were noticed in CYP+MS- treated rats (Fig. 5 C). In CYP+RGH- treated rats (Fig. 5 D) and CYP+MS+RGH treated rats (Fig. 5 E) there was an obvious distinction between the white and the red pulp, the white pulp had normal accumulation of cellularity and the red pulp showed an increase in the number of the reticular cells and macrophages and most sections of spleen showed an apparently normal structure for white and red pulps as it was observed in the normal control sections.
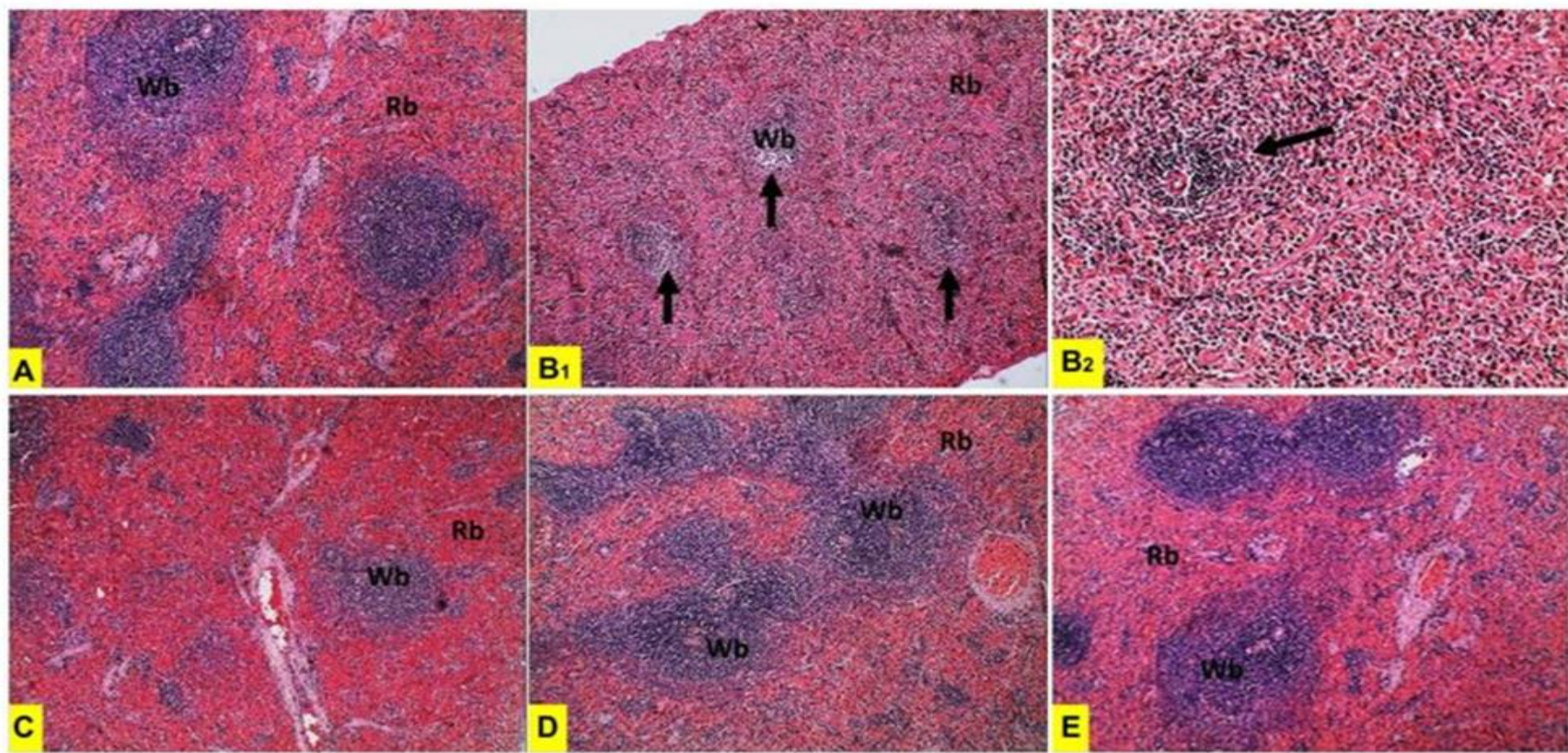

Figure 5 Photomicrographs of spleen sections of rats in different groups (H\&E stain X4 ; B2 X10) (Wb: white bulb, Rb: red bulb, FA: follicular arteriole, arrow: depletion in white bulb) A: Control group showing: Normal spleen architecture. B: CYP group showing dispersed lymphatic nodules with decreased cellularity. C: CYP+MS group, D: CYP+RGH group, E: CYP+MS+RGH group showing somewhat normal structure for white and red pulps.

\section{Discussion}

Cyclophosphamide is an alkylating agent that is commonly used as an antineoplastic and immunosuppressive drug. Notwithstanding its chemotherapeutic properties, CYP causes oxidative systemic damages [26]. Mesna has been widely used as an effective drug against CYP adverse effects. Recently, several studies applied to study the potential ameliorative role of natural remedies to minimize chemotherapeutics toxicity without affecting their antineoplastic activities. Therefore, this study aimed to investigate the potential role of raw garlic homogenate for improving the protective effect of mesna against cyclophosphamide toxicity in rats.

Our findings revealed that single i.p. administration of CYP at a dose of $200 \mathrm{mg} / \mathrm{kg}$ b.wt. significantly decreased the levels of RBCs, Hb, PCV without any significant changes in MCV, MCH and MCHC along with thrombocytopenia. Moreover, CYP- intoxicated rats showed significant reduction in TLC, neutrophils and lymphocytes with insignificant reduction in eosinophils, basophils and monocytes. 
The above hematological findings were in agreement with Cengiz [27] and Elshater et al. [28] who stated that administration of $200 \mathrm{mg} / \mathrm{kg}$ of CYP to rat resulted in reductions in the number of erythrocyte, hemoglobin, leukocytes, thrombocytes and hematocrit. Also, administration of 50,100, or $150 \mathrm{mg} / \mathrm{kg}$ of CYP, i.p. caused, in a dose-dependent manner, reductions in the number of RBCs, WBCs and platelets. It is well known that CYP adversely affects the hematopoietic and immune systems leads subsequently to further hematopoietic and immune dysfunction, which represented by anemia, thrombocytopenia and leukopenia [29].

Regarding the effect of CYP on oxidant/ antioxidant status, our findings showed that CYP induced oxidative damage as evidenced by significant increase in serum MDA content concomitantly with reduction in TAC after single i.p. injection of CYP. Inconsistent with our findings, Gunes et al. [30] recorded that rats treated with $100 \mathrm{mg} / \mathrm{kg}$ CYP induced significant increase in serum MDA with reduction in TAC. Moreover, previous investigations recorded the tissue oxidative damages of CYP as demonstrated by increase of MDA and reduction of antioxidants [28, 31-35].

Oxidative stress is the imbalance of oxidant/antioxidant state leading to oxidation of lipids, DNA and proteins in the cells and hence, tissue damage. Lipid peroxidation is an oxidative destruction of poly unsaturated fatty acid. MDA is one of end products of lipid peroxidation and is used to access lipid peroxidation and free radical generation along with the estimation of TAC. Oxidative stress and generation of reactive oxygen species (ROS) have been implicated in the pathophysiology of CYP toxicity [36]. CYP induces oxidative damage through decreasing the activities of the antioxidant enzymes and increases the extent of the lipid peroxidation [37].

The mechanism by which CYP induces tissue damages could be attributed it to its cytotoxic metabolites; phosphoramide mustard and acrolein. Phosphoramide mustard exerts antineoplastic effect while acrolein, is a highly reactive metabolite that causes oxidative stress implicated in the urotoxic, hepatotoxic, nephrotoxic, cardiotoxic and immunotoxic effects of CYP [28,30-32,34,38]. Also, CYP cytotoxicity may be due to the production of TNF- $\alpha$, a pleiotropic cytokine inducing cell death via necrosis and apoptosis pathway [39].

The recorded oxidative biochemical changes were supported by the observed histopathological alterations of the examined tissues. The same histopathological findings were previously observed in urinary bladder [32], liver [28], kidney [35], heart [34] and spleen [31] of CYP- treated rats.

The obtained results approved the cytoprotective effect of mesna and/ or garlic against CYP toxicity that evidenced by improvement of the altered hematological, biochemical and histopathological findings with marked improvement in rats administrated a combination of mesa and garlic. In agreement with our findings, previous studies reported the protective effect of mesna [40] and garlic [41, 42] against CYP toxicity.

Mesna has been routinely used with CYP to alleviate its side effects mainly the hemorrhagic cystitis [43]. Acrolein binding and ROS scavenging properties of MS may contribute to its beneficial protective and therapeutic properties [44, 45]. Şener et al. [45] suggested that mesna, as an antioxidant and thiol-containing drug, exhibited hepatorenal protective effect against acetaminophen-induced oxidative damage. Moreover, Şener et al. [46] stated that mesna with its antioxidant and antifibrotic properties may be of potential therapeutic value for protecting the liver against biliary obstruction-induced oxidative damage. Consequently, the recorded cytoprotective effect of mesna could be attributed to its antioxidant property.

Garlic, a natural dietary substance, has been well recognized for its medicinal properties [47]. Garlic and its compounds exert antimicrobial, anti-inflammatory, anti-artherosclerotic, antihypertensive and antihyperlipidemic, antidiabetic, hepato-renoprotective, antioxidant, anti- carcinogenic and immune modulation, and various other biological properties [18]. Previous studies have reported the protective effect of garlic against doxorubicin [48], cadmium [49], acrylamide [50] and cisplatin [51] toxicities. The beneficial therapeutic value of garlic against different toxic agents was mainly via its powerful antioxidant and free radical scavenging properties [18, 50]. RGH contains numerous antioxidant organosulfur compounds, mainly S-Allylcysteine and allicin. These compounds play an important role as antioxidants [52] and exert their antioxidant actions by inhibiting lipid peroxidation, scavenging ROS, enhancing cellular antioxidant enzymes and increasing glutathione in the cells [18]. Our findings approved the immune-stimulant and the antioxidant properties of garlic. 


\section{Conclusion}

From these observations, it can be concluded that administration of mesna or garlic alone may be insufficient in the management or treatment of the various adverse effects induced by cyclophosphamide in rats. On the other hand, administration of garlic in combination with mesna completely alleviated cyclophosphamide adverse effects. Therefore, garlic supplementation in combination with mesna could be of a great value to introduce therapeutic strategies for patients undertaking cyclophosphamide therapy.

\section{Compliance with ethical standards}

\section{Acknowledgments}

All authors would gratefully thank the team of Central Lab, Faculty of Veterinary Medicine, University of Sadat City for their technical support in the implementation of this study.

\section{Disclosure of conflict of interest}

The author declares no conflicts of interest. The author had no financial grants or external fund.

\section{Statement of ethical approval}

This study was ethically approved by the International Animal Care and Use Committee (IACUC), Faculty of Veterinary Medicine, University of Sadat City.

\section{References}

[1] Matz EL and Hsieh MH. (2017). Review of Advances in Uroprotective Agents for Cyclophosphamide- and Ifosfamide-induced Hemorrhagic Cystitis. Urology, 100, 10-16.

[2] Morandi P, Ruffini PA, Benvenuto GM, Raimondi R and Fosser V. (2005). Cardiac toxicity of high-dose chemotherapy. Bone Marrow Transplant, 35, 323-334.

[3] Uber WE, Self SE, Van Bakel AB and Pereira NL. (2007). Acute antibody mediated rejection following heart transplantation. Am J Transplant, 7(9), 2064-2074.

[4] Perini P, Calabrese M, Rinaldi L and Gallo P. (2008). Cyclophosphamide-based combination therapies for autoimmunity. Neurological Science, 29, 233-234.

[5] Suwa A, Hirakata M, Satoh S, Mimori T, Utsumik K and Inada S. (1999). Rheumatoid arthritis associated with methotrexate-induced pneumonitis: Improvement with cyclophosphamide therapy. Clinical Experimental Rheumatology, 17, 355-358.

[6] Sharda SV, Gulati S, Tripathi G, Jafar T, Kumar A, Sharma RK and Agrawal S. (2008). glutathione-S-transferase polymorphisms influence response to intravenous cyclophosphamide therapy in idiopathic nephrotic syndrome? Pediatric Nephrology, 23, 2001-2006.

[7] Hu RQ, Mehter H, Nadasdy T, Satoskar A, Spetie DN and Rovin BH. (2008). Severe hemorrhagic cystitis associated with prolonged oral cyclophosphamide therapy: case report and literature review. Rheumatology International, $28,1161-4$.

[8] Viswanatha-Swamy, AH, Patel, UM, Koti, BC, Gadad, PC, Patel, NL and Thippeswamy, AH. (2013). Cardioprotective effect of Saraca indica against cyclophosphamide induced cardiotoxicity in rats: A biochemical, electrocardiographic and histopathological study. Indian Journal of Pharmacology, 45(1), 44-48.

[9] Conklin DJ, Haberzettl P, Jagatheesan G, Baba S, Merchant ML, Prough RA, Williams JD, Prabhu, SD and Bhatnagar A. (2015). Glutathione S-transferase P protects against cyclophosphamide-induced cardiotoxicity in mice. Toxicology and Applied Pharmacology, 285(2), 136-148.

[10] Amien AI, Fahmy SR, Abd-Elgleel FM and Elaskalany SM. (2015). Renoprotective effect of Mangifera indica polysaccharides and silymarin against cyclophosphamide toxicity in rats. The Journal of Basic \& Applied Zoology, $72,154-162$. 
[11] Khorwal G, Chauhan R and Nagar M. (2017). Effect of cyclophosphamide on liver in albino rats: A comparative dose dependent histomorphological study. International Journal of Biomedical and Advance Research, 8(03), 102-107.

[12] Kyung YS, Park HY and Lee G. (2011). Preservation of uroplakins by 2-mercaptoethanesulfonate in cyclophosphamide-induced rat cystitis. Archives of Toxicology, 85, 51-57.

[13] Triantafyllidis T, Taitzoglou I, Kesisoglou I, Lazaridis C and Botsios D. (2015). Treatment with Mesna and n-3 polyunsaturated fatty acids ameliorates experimental ulcerative colitis in rats. International Journal of Experinmental Pathology, 96(6), 433-443.

[14] Sak K. (2012). Chemotherapy and Dietary Phytochemical Agents. Chemotherapy Research and Practice, 2012, Article ID 282570, 11 pages.

[15] Petrovic V, Nepal A, Olaisen C, Bachke S, Hira L, Søgaard CK, Røst LM, Misund K, Andreassen T, Melø TM, Bartsova Z, Bruheim P and Otterlei M (2018). Anti-Cancer Potential of Homemade Fresh Garlic Extract Is Related to Increased Endoplasmic Reticulum Stress. Nutrients, 10(4), 450.

[16] El-Sheshtawy SM, El-Keredy MS and Eltalawy MF. (2016). Antioxidant Potential and Toxicity of Garlic (Allium sativum). Egyptian Journal of Chemistry and Environmental Health, 2 (2), 56-65.

[17] Josling PA. (2005). The heart of garlic Nature's aid to healing the human body, HEC Publishing, Chicago Illinois, 20.

[18] Capasso A. (2013). Antioxidant action and therapeutic efficacy of allium sativum L. Molecules, 18(1), 690-700.

[19] Douaouya L. and Bouzerna N. (2016). Effect of garlic (allium sativum l) on biochemical parameters and histopathology of pancreas of alloxan-induced diabetic rats. International Journal of Pharmacy and Pharmaceutical Sciences, 8 (6), 202-206.

[20] Şengül E, Gelen V, Gedikli S, Özkanlar S, Cur S and Çınar A. (2017). The protective effect of quercetin on cyclophosphamide-Induced lung toxicity in rats. Biomedicine \& Pharmacotherapy, 92, 303-307.

[21] Kanat O, Kurt E, Yalcinkaya U, Evrensel T and Manavoglu O. (2006). Comparison of roprotective efficacy of mesna and amifostine in Cyclophosphamide- induced hemorrhagic cystitis in rats. Indian Journal of Cancer, 43(1), 1215.

[22] Weiss DJ and Wardrop KJ. (2010). Schalm's Veterinary Hemetology. New York, Blackwell, $6^{\text {th }}$ ed.

[23] Ohkawa H, Ohishi W and Yagi K. (1979). Assay for lipid peroxides in animal tissues by thiobarbituric acid reaction. Analytical Biochemistry, 95, 351-358.

[24] Koracevic D, Koracevic G, Djordjevic V, Andrejevic S and Cosic V. (2001). Method for the measurement of antioxidant activity in human fluids. J. Clin. Pathol.54, 361-356.

[25] Bancroft JD and Gamble M. (2008). Theory and Practice of histological techniques". 6th Edition. Churchill, Livingstone, New York, London. 440-450.

[26] Eolayinka ET, Ore A, Ola OS and Adeyemo OA. (2015). Ameliorative Effect of Gallic Acid on CyclophosphamideInduced Oxidative Injury and Hepatic Dysfunction in Rats. Medical Science (Basel), 3(3), 78-92.

[27] Cengiz M. (2018). Hematoprotective effect of boron on cyclophosphamide toxicity in rats. Cellular and Molecular Biology, 64(5), 62-65.

[28] Elshater AA, Haridy MAM, Salmana MMA, Fayyada AS and Hammad S. (2018). Fullerene C60 nanoparticles ameliorated cyclophosphamide-induced acute hepatotoxicity in rats. Biomedicine \& Pharmacotherapy, 97, 5359.

[29] Vadhan-Raj S. (2009). Management of chemotherapy-induced thrombocytopenia: current status of thrombopoietic agents. Seminars in Hematology, 46(1-2), S26-32.

[30] Gunes S, Ayhanci A, Sahinturk V, Altay D and Uyar R. (2017). Carvacrol attenuates cyclophosphamide-induced oxidative stress in rat kidney. Canadian Journal of Physiology and Pharmacology, 95 (7), 844-849.

[31] Hermenean A, Ardelean A and crăciun C. (2008). Spleen Structural Damages and Tissular Biochemical Parameters Disturbances at Wistar Rats after Chemotherapy Administration. Life Sciences Series, 18, $255-258$. 
[32] Tasdemir S, Tasdemir C, Vardi N, Ates B, Taslidere E, Karaaslan MC, Sapmaz HI, Sagir M, Kurt A and Baser CA. (2013). Effects of Ozone therapy on Cyclophosphamide-induced Urinary Bladder Toxicity in Rats. Clinical and Investigative Medicine, 36 (1), E9-E17.

[33] Ahmed LA, EL-Maraghy SA, and Rizk SM. (2015). Role of the KATP channel in the protective effect of nicorandil on cyclophosphamide-induced lung and testicular toxicity in rats.

[34] Omole JG, Ayoka OA, Alabi QK, Adefisayo MA, Asafa MA, Olubunmi BO and Fadeyi BA. (2018). Protective Effect of Kolaviron on Cyclophosphamide-Induced Cardiac Toxicity in Rats. Journal of Evidence-Based Integrative Medicine, 23, 1-11.

[35] Bokolo B and Adikwu E. (2018). Protective assessment of cimetidine against cyclophosphamide-induced kidney injury. Asian Journal of Medical Sciences, 9 (6), 25-30.

[36] Motawi TM, Sadik NA and Refaat A. (2010). Cytoprotective effects of DL alpha- lipoic acid or squalene on cyclophosphamide-induced oxidative injury: an experimental study on rat myocardium, testicles and urinary bladder. Food and Chemical Toxicology, 48(8-9), 2326-2336.

[37] Haque R, Bin-Hafeez B, Parvez S, Pandey S, Sayeed I, Ali M and Raisuddin S. (2003). Aqueous extract of walnut (Juglans regia L.) protects mice against cyclophosphamide induced biochemical toxicity. Human and Experimental Toxicology, 22, 473-80.

[38] Honjo T, Suou T and Hirayama C. (1988). Hepatotoxicity of cyclophosphamide in man: pharmacokinetic analysis. Research Communication in Chemical Pathology and Pharmacology, 61(2), 149-165.

[39] Wu X, Guo R, Chen P, Wang Q and Cunningham PN. (2009). TNF induces caspase-dependent inflammation in renal endothelial cells through a Rho and myosin light chain kinase dependent mechanism. American Journal of Physiology and Renal Physiology, 297, 316-326.

[40] Ozcan A, Korkmaz A, Oter S and Coskun O. (2005). Contribution of flavonoid antioxidants to the preventive effect of mesna in cyclophosphamide- induced cystitis in rats. Archives Toxicology, 79(8), 461-5.

[41] Ahmed RA. (2018). Hepatoprotective and antiapoptotic role of aged black garlic against hepatotoxicity induced by cyclophosphamide. The Journal of Basic and Applied Zoology, 79 (8), 1-8.

[42] El-Sebaey AM, Abdelhamid FM and Abdalla OA. (2019). Protective effects of garlic extract against hematological alterations, immunosuppression, hepatic oxidative stress, and renal damage induced by cyclophosphamide in rats. Environmental Science and Pollution Resarch.

[43] Shepherd JD, Pringle LE, Barnett MJ, Klingemann HG, Reece DE and Phillips GL. (1991). Mesna versus hyperhydration for the prevention of cyclophosphamide-induced hemorrhagic cystitis in bone marrow transplantation. Journal of Clinical Oncology, 9(11), 2016-2020.

[44] Mashiach E, Sela S, Weinstein T, Cohen HI, Shasha SM and Kristal B. (2001). Mesna: A novel renoprotective antioxidant in ischaemic acute renal failure. Nephrology Dialysis Transplantation, 16, 542-551.

[45] Şener G, Sehirli Ö, Cetinel S, YeSen BG, Gedik N and Dülger A. (2005). Protective effects of MESNA (2mercaptoethane sulphonate) against acetaminophen-induced hepatorenal oxidative damage in mice. Journal of Applied Toxicology, 25, 20-29.

[46] Şener G, Kabasakal L, Sehirli O, Ercan F and Gedik N. (2006). 2-Mercaptoethane sulfonate (MESNA) protects against biliary obstruction-induced oxidative damage in rats. Hepatology Research, 35(2), 140-6.

[47] Bongiorno PB, Fratellone PM and LoGiudice P. (2008). Potential health benefits of garlic (Allium sativum): a narrative review. Journal of Complementary Integrative Medicine, 5(1), 1-26.

[48] Alkreathy H, Zoheir AD, Nessar A, Mark S, Soad SA and Osman AM. (2010). Aged garlic extract protects against doxorubicin-induced cardiotoxicity in rats. Food and Chemical Toxicology, 48, 951-956.

[49] Lawal AO and Ellis EM. (2011).The chemopreventive effect of aged garlic extract against cadmium-induced toxicity. Environmental Toxicology and Pharmacology, 32, 266-274.

[50] Abd El-Halim SS and Mohamed MM. (2012). Garlic powder attenuates acrylamide-induced oxidative damage in multiple organs in Rat. Journal of Applied Sciences Research, 8(1), 168-173.

[51] Nasr AY and Saleh HAM. (2014). Aged garlic extract protects against oxidative stress and renal changes in cisplatin-treated adult male rats. Cancer Cell International, 28(14), 92. 
Tahoun et al. / GSC Biological and Pharmaceutical Sciences 2019, 07(03), 008-020

[52] Colin-Gonzalez AL, Santana RA, Silva-Islas CA, Chanez-Cardenas ME, Santamaria A and Maldonado PD. (2012).The antioxidant mechanisms underlying the aged garlic extract- and S-allylcysteine-induced protection. Oxidative Medicine and Cellular Longevity, 907, 162-78.

\section{How to cite this article}

Tahoun EA, El- Borai NB, Khalifa HK and El- Gendy H. (2019). Contribution of garlic for improving the cytoprotective effect of mesna against cyclophosphamide toxicity in rats. GSC Biological and Pharmaceutical Sciences, 7(3), 08-20. 